\title{
Corrigendum: A Novel Variation in the Mitochondrial Complex I Assembly Factor NDUFAF5 Causes Isolated Bilateral Striatal Necrosis in Childhood
}

\begin{abstract}
Hongyan $\mathrm{Bi}^{1 *}$, Hui Guo ${ }^{2}$, Qianfei Wang ${ }^{3}$, Xiao Zhang ${ }^{1}$, Yaming Zhao ${ }^{1}$, Jimei $\mathrm{Li}^{1}$, Weiqin Zhao ${ }^{1}$, Houzhen Tuo ${ }^{1}$ and Yongbo Zhang ${ }^{1}$

${ }^{1}$ Department of Neurology, Beijing Friendship Hospital, Capital Medical University, Beijing, China, ${ }^{2}$ Center for Medical Genetics and Hunan Key Laboratory of Medical Genetics, School of Life Sciences, Central South University, Changsha, China, ${ }^{3}$ CAS Key Laboratory of Genomic and Precision Medicine, Collaborative Innovation Center of Genetics and Development, Beijing Institute of Genomics, Chinese Academy of Sciences (CAS), Beijing, China
\end{abstract}

Keywords: bilateral striatal necrosis, NDUFAF5, mitochondrial complex I deficiency, whole-exome sequencing, novel variation

\section{A Corrigendum on}

A Novel Variation in the Mitochondrial Complex I Assembly Factor NDUFAF5 Causes Isolated Bilateral Striatal Necrosis in Childhood

by Bi, H., Guo, H., Wang, Q., Zhang, X., Zhao, Y., Li, J., Zhao, W., Tuo, H., and Zhang, Y. (2021). Front. Neurol. 12:922. doi: 10.3389/fneur.2021.675616

Error in Table

On a recent occasion, we realized that in the original article, there was a mistake in Table $\mathbf{1}$ as published. The citation numbers in the Table 1 referring to the NDUFAF5 mutations in various ethnic groups did not match the given reference order list in the published article. In Table 1, (1) reference 26 should be reference (1); (2) reference 27 should be reference 30; (3) reference 28 should be reference (2); (4) reference 12 should be reference 31 ; (5) reference 29 should be reference 32; and (6) reference 13 should be reference (3). The corrected Table 1 appears below.

\section{Missing Citation}

In the original article References 30, 31, and 32 were not cited/included in the published article. The citation has now been inserted in Table 1, under the section Discussion.

New References to be Added in the continuing order:

30. Fang F, Shen Y, Shen DM, Liu ZM, Ding CH, Zhang WC, et al. [Clinical and genetic characteristics of children with Leigh syndrome]. Zhonghua er ke za zhi = Chinese J Pediatr. (2017) 55:205-9. doi: 10.3760/cma.j.issn.0578-1310.2017.03.008

31. Tong W, Wang Y, Lu Y, Ye T, Song C, Xu Y, et al. Whole-exome sequencing helps the diagnosis and treatment in children with neurodevelopmental delay accompanied unexplained dyspnea. Sci Rep. (2018) 8:5214.

32. Gerards M, Sluiter W, van den Bosch BJ, de Wit LE, Calis CM, Frentzen M, et al. Defective complex I assembly due to C20orf7 mutations as a new cause of Leigh syndrome. J Med Genet. (2010) 47:507-12. doi: 10.1136/jmg.2009.067553

The authors apologize for this error and confirm that it does not change the scientific conclusions of the article in any way. The original article has been updated. 
TABLE 1 | Clinical features of patients with NDUFAF5 variations reported in literature.

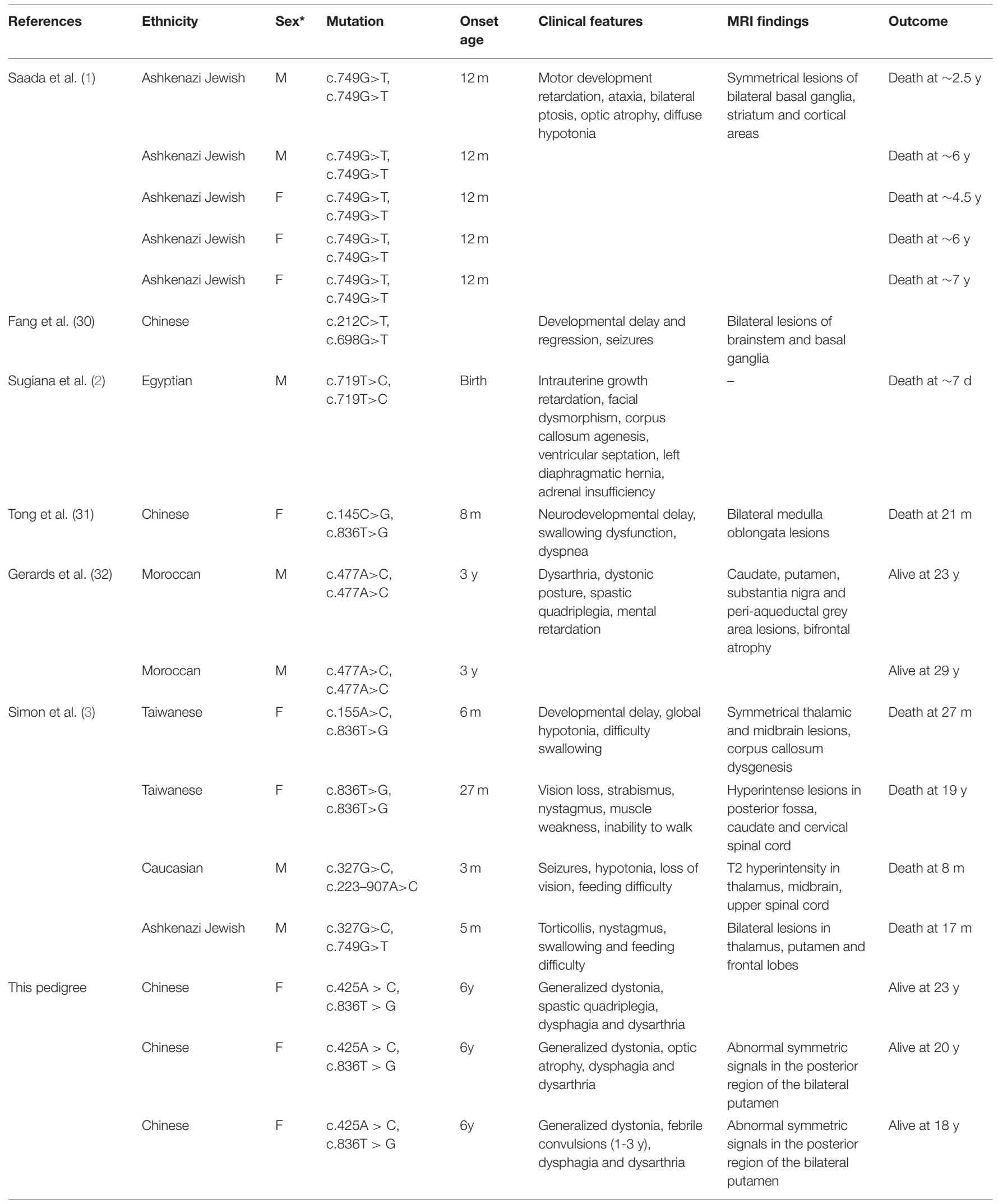

"M, Male; F, Female. 


\section{REFERENCES}

1. Saada A, Edvardson S, Shaag A, Chung WK, Segel R, Miller C, et al. Combined oxphos complex $\mathrm{i}$ and iv defect, due to mutated complex i assembly factor c20orf7. J Inherit Metab Dis. (2012) 35:125-31. doi: 10.1007/s10545-011-9348-y

2. Sugiana C, Pagliarini DJ, McKenzie M, Kirby DM, Salemi R, Abu-Amero $\mathrm{KK}$, et al. Mutation of c20orf7 disrupts complex $\mathrm{i}$ assembly and causes lethal neonatal mitochondrial disease. Am J Hum Genet. (2008) 83:468-78. doi: 10.1016/j.ajhg.2008.09.009

3. Simon MT, Eftekharian SS, Stover AE, Osborne AF, Braffman BH, Chang $\mathrm{RC}$, et al. Novel mutations in the mitochondrial complex i assembly gene ndufaf5 reveal heterogeneous phenotypes. Mol Genet Metab. (2019) 126:53-63. doi: 10.1016/j.ymgme.2018.11.001
Publisher's Note: All claims expressed in this article are solely those of the authors and do not necessarily represent those of their affiliated organizations, or those of the publisher, the editors and the reviewers. Any product that may be evaluated in this article, or claim that may be made by its manufacturer, is not guaranteed or endorsed by the publisher.

Copyright (c) 2021 Bi, Guo, Wang, Zhang, Zhao, Li, Zhao, Tuo and Zhang. This is an open-access article distributed under the terms of the Creative Commons Attribution License (CC BY). The use, distribution or reproduction in other forums is permitted, provided the original author(s) and the copyright owner(s) are credited and that the original publication in this journal is cited, in accordance with accepted academic practice. No use, distribution or reproduction is permitted which does not comply with these terms. 